\title{
K-coverage Algorithm Based on Target Association in Wireless Sensor Network
}

\author{
Zeyu Sun \\ Department of Computer and Information Engineering, \\ Luoyang Institute of Science and Technology \\ Luoyang, 471023, China
}

\author{
Chuanfeng Li \\ Department of Computer and Information Engineering, \\ Luoyang Institute of Science and Technology \\ Luoyang, 471023, China
}

\begin{abstract}
During the process of carrying out $\mathrm{K}$ coverage algorithm of WSN to the target area, there are some shortcomings, such as many redundancy nodes will emerge, the process consumes a lot of node energy in the network at the same time, and the process is easily restricted by the outside environmental elements. Countering the shortcomings, this article puts forward an event probability $K$ coverage algorithm. By means of giving different probability value to different monitor degree of the target area nodes, and by way of information exchange and relevance nature between the nodes, the algorithm fixes the smallest nodes collection and the biggest target collection, thus effectively fulfills $K$ coverage to target area nodes, optimizes network resources, reduces energy consumption of the nodes, prolongs the life time of the network. Simulation experiments show that, this algorithm can fulfill $K$ coverage to target area at small cost and increases the whole network life time, has good practical result and stability.
\end{abstract}

Keywords-Wireless sensor network (WSN), Event probability, Coverage, Energy

\section{INTRODUCTION}

Wireless sensor networks (Wireless Sensor Network, WSN) combines the embedded technology, wireless communication technology, distributed technology, and could collecting, computing, communicating, processing data successively. While, little size and finite energy of nodes limit its application. In present, many scholars have carried out in-depth study and discussion on this issue. And his problem is attracting much attention. LIU [1] presented the solving methods for partial coverage, and proposed that in some extent partial coverage is superior to complete coverage to save the energy of the network. However, the premise of this method is the incompleteness coverage of the target monitoring area. There is no discussion about how to achieve optimal coverage algorithm for partial coverage and how to maintain network connectivity. Xing [2] studied the connectivity issues and presented SPAN + CCP program which for one degree connectivity and this program is based on the communication radius of nodes in wireless sensor network less than twice the induction radius. There is no solution about multi-degree connectivity. Above all, there are many problems restricted the life time of the network.

This Algorithm giving different probability value to different monitor degree of the target area nodes, determining the smallest nodes collection and the biggest target collection through the exchange of information between the nodes and the associated attributes, deploying the node at different state through the state transitions mechanism between nodes, then the network resources was optimized, energy consumption of the nodes was reduced and the life time of the network was prolonged.

\section{PROBLEM DEFINITION}

\section{A. DEFINITIONS}

Definition 1: Area A is covered by the si node means that any point $\mathrm{p}$ in the area Asi is under the coverage of the si node.It can be described as following:

$$
A s_{i}=\left\{p \mid d(p,(x, y)) \leq r_{s}, i \in[1, n]\right\}
$$

Where $\mathrm{d}$ is the Euler distance between two points, ri is the induction radius of nodes, $\mathrm{n}$ is the number of nodes [3].

Definition 2: Given the planar A, the coordinates of the node si $\left(x_{i}, y_{i}\right)$, the coordinates of the target $t_{k}\left(x_{k}, y_{k}\right)$, the Euler distance between $S_{i}$ and $t_{k}$ less than the induction radius $r_{s}$ of $s_{i}, A S_{i}=\left\{t_{k} \mid t_{k} \in A^{2}, D\left(s_{i}, t_{k}\right) \leq r_{s}\right\}$, So the $t_{k}$ is covered by the ${ }^{s_{i}}$. Given the target set $\mathrm{T}=\{\mathrm{t} 1, \mathrm{t} 2, \mathrm{t} 3 \ldots \mathrm{tk}\}$ and the sensor node set $\mathrm{V}=\{\mathrm{v} 1, \mathrm{v} 2, \mathrm{v} 3 \ldots \mathrm{vn}\}$, In a time slot, if one destination node tk within the target set is covered at least by one vn node in the sensor node set, called for complete coverage of target set $\mathrm{T}$.

Definition 3: The coverage ratio for one node si in the

region is:

$$
p\left(s_{i}, s_{j}\right)= \begin{cases}0 & \text { if } \mathrm{r}_{s} \leq d\left(u, s_{i}\right) \\ e^{-\varepsilon d} & \text { if }\left(\mathrm{r}_{s}-r_{e}\right)<d\left(s_{i}, s_{j}\right)<r \\ 1 & \text { if } \mathrm{d}\left(\mathrm{s}_{i}, \mathrm{~s}_{j}\right) \leq r_{s}-r_{e}\end{cases}
$$

Where: $d=d\left(s_{i}, s_{j}\right)-\left(r_{s}-r_{e}\right), \quad \varepsilon$ is the impact factor, indicate physical parameters. re is the uncertain factor in the monitoring process. $d\left(s_{i}, s_{j}\right)$ is the Euler distance between 
si and $\mathrm{sj}$. When $\mathrm{p} \geq \mathrm{pth}$, si is covered, otherwise it is not been covered. pth is the coverage ratio of si when it meet some cover.

\section{B. NETWORK MODELING}

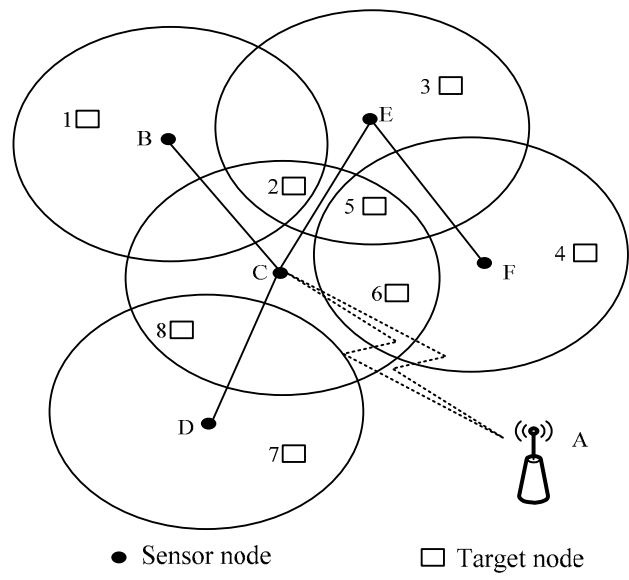

Figure 1. Network modeling

If there is a data exchange between si and $\mathrm{sj}$, the two adjacent nodes to be clusters according to the distribution of local information. And nodes in cluster will be cluster head in each network cycle in turn [4]. Cluster head node using TDMA scheduling to complete the communication between the cluster nodes, and the cluster head node fusion processing the received data, then the results are forwarded to the integration of convergence node through the shortest path tree which is established based on the cluster head node. As shown in Figure.1.

The lower layer is the sensor layer, which are combined by five nodes. They collect data of different types, and constitute some cluster structures according to certain rules. The upper layer is the Sinks layer [5]. Such as: sensor node $\mathrm{B}$ and the destination node 1,2 ; sensor node $\mathrm{C}$ and the destination node 2,5,6,8; sensor node $\mathrm{D}$ and the destination node 7,8; sensor node $\mathrm{E}$ and the destination node 2,3,5; sensor node $\mathrm{F}$ and the destination node 3,4,5,6. According to the association attribute, the smallest set of nodes $S=\{B, D$, F $\}$ and the maximum target set $T=\{3,4,5,6\}$ are defined. When the sensor nodes increased, a target node will be covered by $\mathrm{K}$ sensor nodes. This is the $\mathrm{K}$ Coverage. It means that a multi-association attribute is formed between one target node and a number of sensor nodes. Such as: the target node 2,5 is covered by $\mathrm{K}=3$ sensor nodes, the same for the target node 6,8 and $\mathrm{K}=2$. When the coverage for a certain target node exceeds its own coverage, large redundant nodes will appear [6].

\section{THEORIES AND ALGORITHMS}

\section{A. ALGORITHMIC}

In the target monitoring area $\mathrm{A}$, the function relation between the number of nodes which covering the entire area A and the radius rs for sensor node is:

$$
n=2 A / 3 r_{s}^{2} \sqrt{3}
$$

At the same time to ensure the number of sensor nodes at least. Where $\mathrm{n}$ is the number of sensor nodes. For each sensor nodes have an event probability, it is assumed that $p=e^{-\varepsilon d} \geq p_{t h}$, then work out the approximate value $r_{s}^{\prime}=-\ln p_{t h} / \varepsilon+r_{s}-r_{e}$ According to the formula (3), and the approximate probability of each sensor node is:

$$
p_{a p p}=2 A / 3 n r_{s}^{\prime 2} \sqrt{3}
$$

The event probability for the node in active state is :

$$
p_{\text {act }}=2 A E_{\text {cur }} / 3 n E_{\text {init }} r_{s}^{\prime 2} \sqrt{3}
$$

Where Ecurr is the current energy, Einit is the initial value of the current energy. Randomly deployed the node in the monitoring region, and constructed a finite set $\mathrm{S}$ using these nodes, so that the coverage probability when $\mathrm{K}$ is the same in the condition $\mathrm{S}$ is not empty is obtained. When the number of nodes $n \rightarrow \infty, \lim _{n \rightarrow \infty} P(S)=1$. It is indicate that when the number of nodes is large enough, the coverage area is completely covered by $\mathrm{K}$ coverage.

\section{B. STEP OF THE ALGORITHM}

Step1: Randomly deploy $N$ sensor nodes in the region of target monitoring. Select the target set $T_{\max }$ and sensor nodes set $S_{\max }$ which has the largest number in the frequent sets. Then choose a high-energy set of sensor nodes from them.

Step2 : Setting the location of sensor nodes and the coverage radius $r$, the entire coverage area is divided into several disjoint regions $\left\{F_{1}, F_{2} \ldots F_{n}\right\}$, each sub-region $F_{i}$ associate the sensor nodes in all coverage area and ensure that included in its scope. That is $F_{l}=\left\{S_{n 1}, S_{n 2} \cdots S_{n l}\right\}$ and $\bigcup_{i=1,2 \cdots n} F_{i}=A$.

Step3 : In order to reduce the amount of overlapping coverage for the sub-region of sparse coverage which induced by the redundant nodes, define as overlap value for $\mathrm{i}$ sensor node.

$v_{i}=F_{c}$ vi is the number of sensor nodes. According to formula (3), it can calculate out that vi is the number of selected important sub-regional which is covered by $i$ sensor node. That is $v_{i}=v_{\min }$. It also is the event probability value of active nodes which is the number of sensor nodes covering least target region, and then calculated through formula (4).

Step4: All the sensor nodes in the current coverage subtract the power which consumed in previous data collection and retrieve from the previous remaining power to update the current remaining power. Denoted by $E_{r}^{i}=E_{r}^{i}-E_{c}^{i}$ If the remaining power of a sensor node is less than the request power for one time data information collected and retrieve, this sensor node will be removed from the set of sensor nodes. 
Step5: When judging the energy of node in target area which is covered by current sensor nodes, $c_{n} \geq w$ Step2 is executed. Until the coverage area A will no longer be covered by available sensor node. At this time, the network lifetime is maximized.

\section{PERFORMANCE EVALUATION}

In this paper, the MATLAB6.5 simulation platform is adopted. The sensor nodes are randomly deployed in the area being $400 \mathrm{~m} \times 400 \mathrm{~m}$. Each sensor has a circular coverage area, its radius is $\mathrm{r}$. Each sensor has a same initial energy is $2 \mathrm{~J}$. The wireless communication models for sensor nodes sending data and receiving data is as follows:

$$
\begin{aligned}
& E_{T r}(k, d)=E_{T-\text { elec }} k+E_{\text {amp }}(k, d) \\
& =\left\{\begin{array}{cc}
E_{T-\text { elec }} k+\varepsilon_{f s} d^{2} k & d<d_{0} \\
E_{T-\text { elec }} k+\varepsilon_{\text {amp }} d^{4} k & d \geq d_{0}
\end{array}\right. \\
& E_{R x}(k)=E_{R-\text { elec }} k
\end{aligned}
$$

In the above formula, ET-elec and ER-elec indicate the energy consumption of module for wireless sending and receiving; \&fs and camp indicate the energy consumption parameters of amplifier for free space model and multichannel attenuation model respectively; $\mathrm{d} 0$ is a constant.

To evaluate the performance, the experiment was carried out in three different conditions. The first, with the same sensor node which is 100 , compared the algorithm we used with the LEACH protocol. Time and events are the same. As shown in Figure.2.

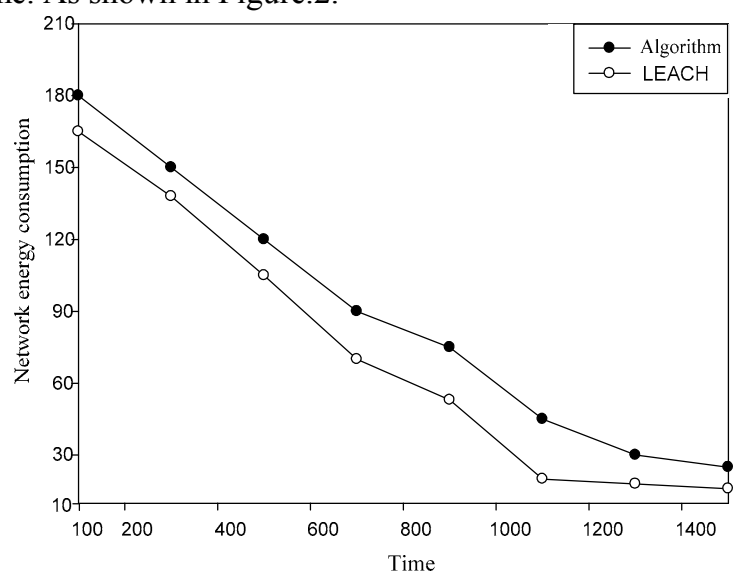

Figure 2. Energy consumption of network

Figure. 2 indicated that the energy consumed by our algorithm is less than LEACH protocol with the same number of rounds. As time prolonged, the energy consumption of Network tends to stable and homogeneous. Compared with the LEACH protocol, it is more energy saving, thus extending the network lifetime.

The second was deployed 200 sensor nodes in the randomly selected area $400 \mathrm{~m} \times 400 \mathrm{~m}$, comparing the number of surviving nodes of LEACH Protocol in the networking process. As shown in Figure.3.
It can be seen from Figure. 3 that the number of survival nodes declined as time prolonged. The number of survival nodes in this algorithm is more than that of the LEACH protocol. All of this indicated that the residual energy of nodes in the algorithm described above is higher than residual energy of nodes in the LEACH protocol, and then extend the network lifetime. Meanwhile, the residual energy of nodes in the algorithm described above is higher than residual energy of nodes in the LEACH protocol at any same times. So the network resources were optimized.

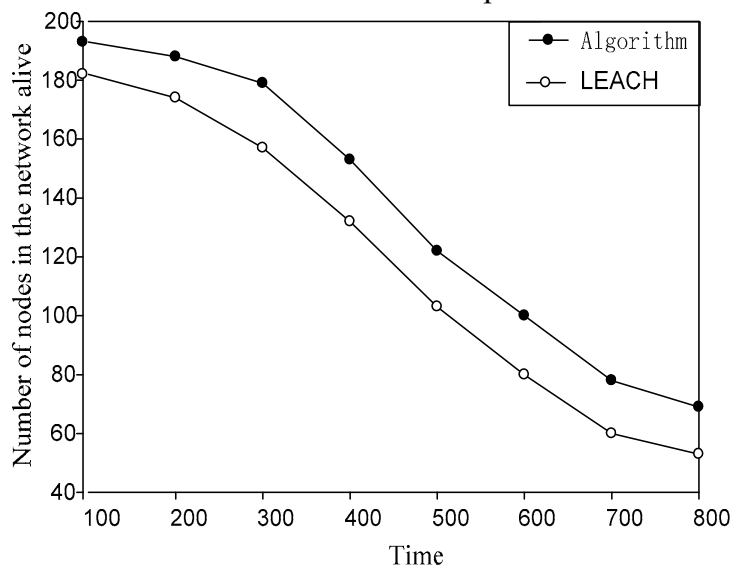

Figure. 3 The number of survival nodes and time

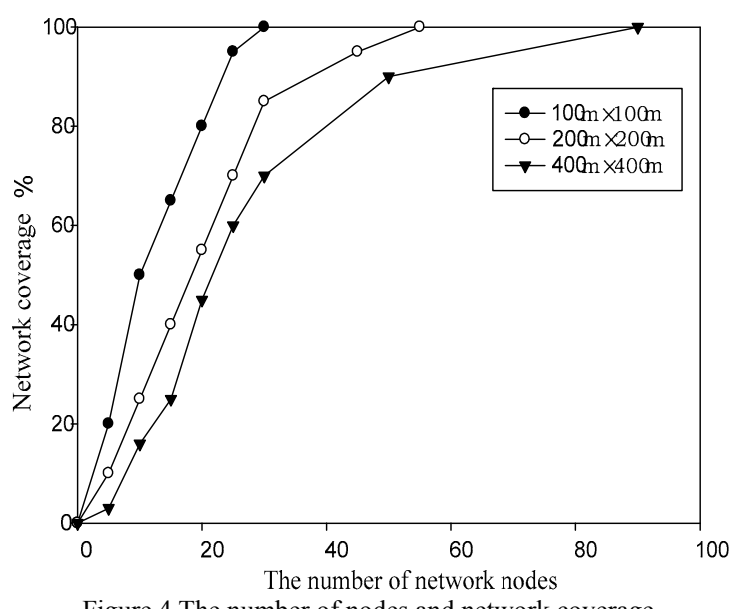

Figure. 4 The number of nodes and network coverage

The third select a different coverage area, $100 \mathrm{~m} \times 100 \mathrm{~m}, 200 \mathrm{~m} \times 200 \mathrm{~m}, 400 \mathrm{~m} \times 400 \mathrm{~m}$, compare the changes of coverage in different areas. The result is shown in Figure.4.

Figure. 4 shows the number of sensor nodes which need to be deployed under different nodes coverage which is achieved at different Network scale. It can be obtained from graph that as the network expansion, the number of nodes needed to be deployed increased to meet the requirement of certain network coverage. The higher the network coverage, the more the number of nodes needed to be deployed. So that to realize the completely coverage of the concerned 
target nodes under the condition of achieve full coverage of target area for different nodes in different coverage area. Within a certain area, the coverage area is different in different area.

\section{SUMMARIES}

Through theoretical analysis of wireless sensor networks, a coverage algorithm was proposed based on event-driven. This algorithm used the association and wakeup mechanism between sensor nodes and target nodes to achieve the coverage for target area. Determined the maximum target set and the minimum node set by the associated attributes between the node and the destination node and optimized. Then reduced the entire network energy consumption and prolonged the network life cycle. Simulation experiments for LEACH algorithms and different coverage area were carried out. The result tested the effectiveness and adaptability of the algorithm.

\section{ACKNOWLEDGMENTS}

This work was supported in part by the Nature Science Foundation Research Project of Henan Province
(2011A590001, 2010B510016), the Funding Scheme for youth teacher of Henan Province (2009GGJS-122).

\section{REFERENCES}

[1] LIU Yu-zhen; LIANG Wei-fa. Approximate Coverage in Wireless Sensor Network, Proceeding of IEEE Conference on Local Computer Networks 30th Anniversary[C], 2005, PP:121-124

[2] XING Guo-liang; WANG Xiao-rui; ZHANG Yuan-fang. Integrated C overage and Connectivity Configuration for Energy Conservation in S ensor Network, ACM Transactions on Sensor Network[J], 2007, PP:36 $-72$

[3] ZHANG Hong-hai; HOU Jennifer. On the upper bound of lifetime for large sensor network, ACM Transanctions on sensor Network[J], 2002, PP:272-279

[4] XING Xiao-fei; WANG Guo-jun; WU Jie et al. Square Region-Based Coverage and Connectivity Probability Model in Wireless Sensor Net works, Proceedings of Collaborate Com-09[C], 2009, PP:1-8

[5] YE Fan; ZHONG Gary; CHENG Jesse; et al. Robust Energy Conservi ng Protocol for long-lived sensor networks, the 23rd Int. Conf. on Dist ributed Computing Systems[C], 2003, PP:28-37

[6] LIU Li-ping; ZHANG Qiang; SUN Yu-geng. Multiple Targets Associ ated Coverage Algorithm in wireless sensor networks, Journal of Tianj in University[J], 2009, PP:483-490 